\title{
Isolation of human small bowel intraepithelial lymphocytes by Annexin V-coated magnetic beads
}

\author{
Francisco León and Garbiñe Roy \\ Department of Immunology, Hospital Ramón y Cajal, Madrid, Spain
}

\begin{abstract}
Human intestinal intraepithelial lymphocytes (IEL) are important effector cells of the mucosal immune system and their study is hampered by the difficulty of their isolation. The molecular study of enriched samples of IEL is mandatory in the diagnosis of enteropathy-associated T-cell lymphoma and refractory celiac sprue. In order to isolate human small bowel IEL, we took advantage of the stress that intestinal epithelial cells (IEC) suffer during the conventional initial steps of IEL isolation, which induces their apoptosis but not that of IEL. After cell individualization by dithiothreitol and ethylenediamine tetraacetic acid, two-thirds of human IEC can be stained with Annexin-V due to their surface exposure of phosphatidyl serine, a sign of apoptosis. This percentage increases to $95 \%$ after performing a density gradient to enrich for IEL. This allows for the use of Annexin-Vcoated magnetic beads, originally designed for the removal of dead cells from cell cultures, to obtain $>95 \%$ pure, $99 \%$ viable and untouched IEL after two rounds of depletion. This simple procedure has proven useful for the isolation of human IEL for functional and molecular studies and can conceivably facilitate the diagnosis of intestinal lymphoid malignancies that rely upon the study of pure IEL preparations.
\end{abstract}

Laboratory Investigation (2004) 84, 804-809, advance online publication, 26 April 2004; doi:10.1038/labinvest.3700099

Keywords: human IEL isolation; Annexin-V; EATL; celiac sprue

Human intraepithelial lymphocytes (IEL) constitute a heterogeneous lymphoid compartment involved in mucosal surveillance against infections and tumors as well as in epithelial growth, immunomodulation and maintenance of oral tolerance. ${ }^{1-3}$ Functional studies with human intestinal IEL are difficult due to the limitations in the collection of normal tissue and in the purification of these cells.

IEL experience profound changes in gluten-sensitive enteropathy or celiac disease, ${ }^{4}$ the most frequent chronic gastrointestinal disorder in children and with a growing prevalence in adults, accounting for $0.5-1 \%$ of the Caucasian population in Europe and the US. ${ }^{5}$ Owing to the continued stimulation of the mucosal immune system, celiac patients may eventually develop refractory celiac sprue (RCS) (a premalignant condition characterized by clonal IEL) and even overt enteropathy-associated T-cell lymphoma (EATL). ${ }^{6}$ The prompt diagnosis of these severe entities require the molecular study of clonal

Correspondence: F León. Current address: Laboratory of Molecular Immunology, NIAID, NIH, 10 Center Drive, Bldg. 10, room 11N214, Bethesda, MD 20892, USA.

E-mail: fleon@niaid.nih.gov

Received 13 November 2003; revised 16 February 2004; accepted 17 February 2004; published online 26 April 2004 rearrangements in the T-cell receptor, ${ }^{6}$ what depends on a sufficient purification of IEL to avoid contamination from other lymphocytes and undesirable amplification of unrelated genetic material.

The most widely used approach to isolate IEL in humans $^{7-9}$ and other species ${ }^{10,11}$ involves several steps: (a) Individualization of epithelial content. The epithelial integrity is disrupted by reducing agents such as dithiothreitol (DTT) and/or cation chelants like ethylene diamine-tetraacetic (EDTA). This liberates IEL and IEC into the medium without altering the basement membrane. If whole murine gut is used, Peyer's patches are removed first. (b) Density gradient $\left(\right.$ Percoll ${ }^{\circledR}$ ) in order to enrich for IEL. Usually, a first centrifugation in $30 \%$ Percoll is performed and then a discontinuous gradient is applied. The usual layers for the second Percoll are $20-44-67 \%$ for human cells ${ }^{7}$ and $30-40 / 45-75 \%$ for mouse or rat cells. ${ }^{10,11}$ This procedure implies a low cell recovery but purity is usually good $(>90 \%$ IEL) ${ }^{10,11}$ though lower for human samples (60-70\%). If higher purity is needed (eg for molecular biology applications), a third step is performed. (c) Purification by immunomagnetic methods or fluorescenceactivated cell sorting. This final step involves staining IEL if their positive selection is preferred (with an antibody such as anti-CD45) or IEC if their 
depletion is favored (with anti-epithelial markers). ${ }^{7}$ In the case of magnetic methods, which offer better viability, anti-Ig-coated paramagnetic beads are generally used to label the cells to be retained in the magnet. Depletion of IEC provides with untouched IEL, and markers such as cytokeratin or BerEP $4^{7}$ have been used for the purpose. However, we have observed that BerEP4 maybe present on IEL, particularly in pathologic conditions (personal observation).

We propose an alternative simple method by using a reagent which is normally utilized to eliminate dead cells from cultures: Annexin-Vcoated paramagnetic beads. During the initial stages of apoptosis, phosphatidyl serine (normally intracellular) is exposed on the surface of cells, allowing for its detection by Annexin-V, that has a high avidity for phosphatidyl serine. The method we propose here takes advantage of this interaction and of the fragility of IEC and has the virtue of rapidly eliminating both IEC and the few nonviable IEL, thus rendering an adequate sample for functional and molecular assays.

\section{Materials and methods}

\section{Mucosal Specimens}

We used four surgical specimens after informed written consent: two were duodenal $\left(4-6 \mathrm{~cm}^{2}\right)$ and were obtained from 'Scopinaro' operations for morbid obesity; two were ileal, obtained from the proximal healthy area of hemicolectomies performed for colonic cancer. This study was approved by the local Ethics Committee of Ramón y Cajal Hospital.

\section{Isolation and Purification of IEL}

Mechanical dispersion of the mucosa

IEL and epithelial cells were extracted by a modification of a described protocol. ${ }^{8,9}$ The specimens were cut into $1-5 \mathrm{~mm}^{2}$ fragments and incubated for $1 \mathrm{~h}$ under intense shaking: the mucosal fragments were placed inside a $50 \mathrm{ml}$ tube contained in a larger tube that was taped to a rotor, so that the former tube was allowed to hit repeatedly the walls of the latter. The medium contained $1 \mathrm{mM}$ DTT and $1 \mathrm{mM}$ EDTA in RPMI 1640 (GibcoBRL Life Technologies, Vienna, Austria) supplemented with 10\% FCS and antibiotics (complete medium or CM). A single cell suspension was obtained by filtering through a $30 \mathrm{~mm}$ sterile filter (Miltenyi Biotec, Bergich Gladbach, Germany), washed in PBS and layered onto the Percoll gradient.

\section{Percoll density gradient}

We used gradients containing 20-40-53-65\% Percoll solution (Amersham Pharmacia, Uppsala, Sweden) in CM. The gradients were spun at
$1900 \mathrm{rpm}$ for $30 \mathrm{~min}$. The cell fraction between $40-$ $53 \%$ Percoll was the most enriched for IEL.

Annexin-bead-based purification of viable IEL

To assess apoptosis, whole epithelial cells and enriched IEL were labeled with FITC-conjugated Annexin-V (Bender, Medsystems, Austria) for $10 \mathrm{~min}$ at RT in HEPES-Ca ${ }^{2+}$ buffer (Medsystems). To isolate viable IEL by means of the depletion of apoptotic and/or dead cells, the enriched IEL fraction from the Percoll was washed in PBS and incubated with Annexin-coated microbeads (Dead Cell Removal kit, Miltenyi Biotec) for $1 \mathrm{~h}$ at RT; the cells were then passed twice through $\mathrm{LD}^{+}$magnetic columns (Miltenyi Biotec) mounted on a MidiMACS magnet (Miltenyi Biotec), according to the manufacturer's protocols.

\section{Flow Cytometric Analysis}

The expression of surface markers on IEL was analyzed by flow cytometry using a FACSCalibur (BD Biosciences, San Diego, CA, USA) after staining with fluorochrome-conjugated mAbs: CD45 (HI30), CD103 (BerACT8), CD19 (4G7), CD8 (SK1) and isotype IgG controls, purchased from $\mathrm{BD}$; and CD4 (T4) from Beckman Coulter (Brea, CA, USA). The data were processed using Lysis II and Cell Quest software (BD), WinMDI (PKWARE Inc, USA) and PRISM (GraphPad Software Inc, USA). Acquisition of multiparameter data was carried out with an appropriate forward scatter (FSC) threshold to exclude erythroid cells and debris. IEL were gated on the basis of their low $90^{\circ}$ light scattering (SSC) and their intraepithelial origin was confirmed by the expression of CD103 and by the absence of CD19 ${ }^{+} \mathrm{B}$ cells in the suspension (Figure 3).

\section{Cytotoxicity Assays}

The lysis of the K562 cell line exerted by purified IEL was measured using a previously described protocol $^{12}$ with minor modifications. Briefly, freshly isolated IEL were incubated with rhIL-2 $(10 \mathrm{ng} / \mathrm{ml})$ (R\&D Systems, Minneapolis, MN, USA) for $12 \mathrm{~h}$ and mixed at the indicated effector:target ratios, centrifuged at low speed $(800 \mathrm{rpm})$ to favor cell contact, and incubated for $4 \mathrm{~h}$. After washing, cells were stained with propidium iodide (PI, Molecular Probes, Eugene, OR, USA). K562 target cells, a human erythroleukemic cell line (American Type Culture Collection, Rockville, MA, USA) were cultured to log phase growth and labeled for the $18 \mathrm{~h}$ prior to the coculture with the lipophilic membrane dye 3,3'-dioctadecyloxacarbocyanine (DiO, Molecular Probes) at $30 \mu \mathrm{M}$. DiO emits green fluorescence and allows for the identification of K562 in the fluorometric biparametric analysis of $\mathrm{DiO} / \mathrm{PI}$ staining. 


\section{Results and discussion}

\section{Annexin-V Staining Ex Vivo}

The mechanical disruption of human small bowel mucosa in the presence of DTT and EDTA yielded 1. $5 \pm 0.4$ million IEL per surgical specimen with a low purity of $4.6 \pm 1.9 \%$ IEL with respect to total cells (Figure 1). In a pilot study of the viability of IEL after dispersion, performed in 15 samples, we observed that the proportion of IEL which were stained by Annexin-V after DTT/EDTA treatment was much lower $(10 \pm 5 \%)$ than that of IEC $(60 \pm 12 \%)$. In addition, the intensity of the staining was substantially higher in IEC (mean fluorescence intensity, MFI, of $700 \pm 75$ ) than in the few Annexin-V ${ }^{+}$IEL (MFI, 70 \pm 13 ) (not shown). After performing a Percoll density gradient, the proportion and intensity of staining of Annexin-V in the enriched IEL fraction $(58.2 \pm 5.1 \%$ IEL in the $40-53 \%$ fraction, Figure 1) was increased, particularly in the contaminating IEC $\left(97 \pm 3 \%\right.$ Annexin- $\mathrm{V}^{+}$enterocytes with $8000 \pm 750 \mathrm{MFI} v s 10 \pm 5 \%$ of Annexin-V ${ }^{+}$-IEL with $1000 \pm 150 \mathrm{MFI}$ ) (Figure 1). We took advantage of this in order to deplete the phosphatidyl-serine ${ }^{+}$ cells.

\section{Isolation of IEL by Annexin-Beads}

The 40-53\% Percoll fractions were incubated with Annexin-coated microbeads for $1 \mathrm{~h}$ (note that this is longer than the manufacturer's standard recommended time) and passed twice through new depletion columns. This procedure provided very high IEL purity $(73.2 \pm 2.2 \%$ after the first and $95.2 \pm 2.8 \%$ IEL after the second depletion) (Figure 2) though low yields ( $<10 \%$ of the initial IEL, $75000 \pm 20000$ IEL in total). The resulting untouched IEL were viable as judged by trypan-blue exclusion (not shown). In addition to the human surgical specimens reported here, we have also utilized this procedure with small bowel biopsies, obtaining a sufficient amount of IEL for molecular biology and functional assays with a minimum of three biopsies. ${ }^{13}$

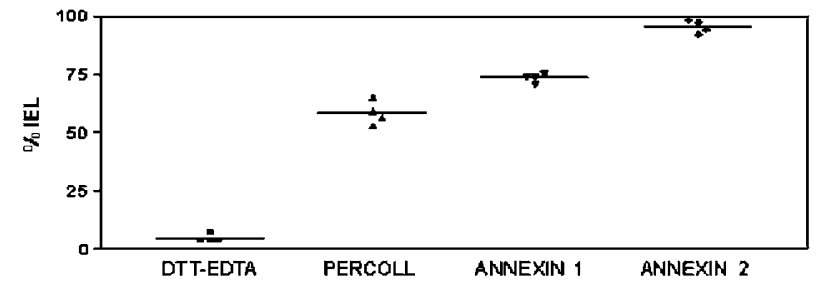

Figure 2 Purity of IEL along the procedure. Purity of IEL in each of four human normal small bowel specimens, in the different steps of the purification: after DTT-EDTA treatment, after the Percoll centrifugation (40-53\% fraction), after the first depletion of Annexin-V + cells ('Annexin 1') and after a second similar depletion ('Annexin 2').

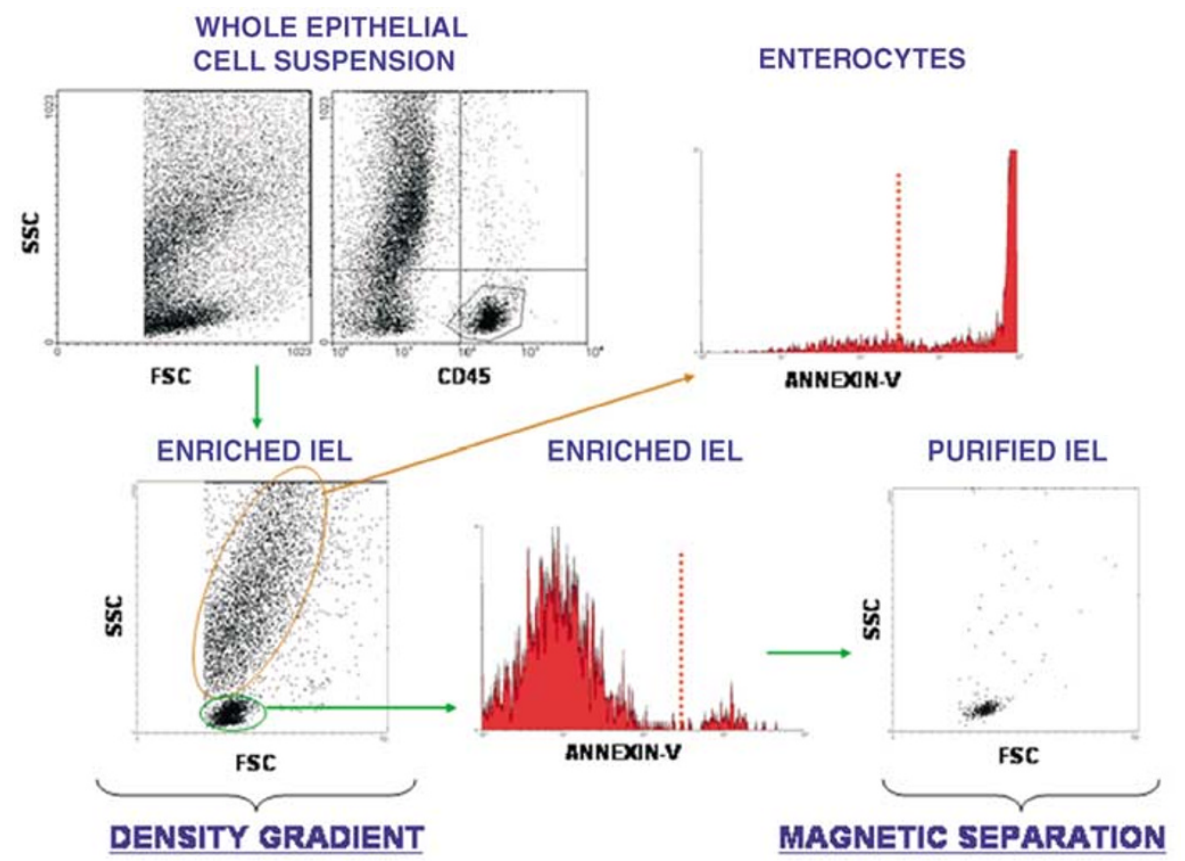

Figure 1 Schematic representation of the isolation procedure. The first two dot plots in the upper left corner illustrate the epithelial mixture of cells ('Whole Epithelial Cell Suspension') obtained after DTT/EDTA treatment of an ileal mucosa specimen. In this representative experiment, the \% of IEL was $7.4 \%$, as can be observed in the CD45/SSC dot plot, where IEL are gated. After Percoll gradient centrifugation, the \% of IEL has increased to $65 \%$ as shown in the 'Enriched IEL' dot plot of the $40-53 \%$ fraction. At this point, $97 \%$ of the IEC are intensely stained with Annexin-V ('Enterocytes' histogram) by only 6\% of the IEL ('Enriched IEL' histogram). After two rounds of magnetic depletion by Annexin-coated beads, the IEL are nearly pure ('Purified IEL' dot plot, 96\% pure and CD45 ${ }^{+}$, not shown). 


\section{Phenotype and Functionality of IEL after Separation}

Human small bowel IEL express the integrin $\alpha \mathrm{E} \beta 7$ (CD103) and comprise two main subsets, CD3 ${ }^{+} \mathrm{T}$ cells and the minoritary CD3 ${ }^{-}$NK subset. ${ }^{13}$ The majority of $\mathrm{CD}^{+}$IEL are CD8 ${ }^{+}$Tc. $^{14}$ The presence of $\mathrm{CD} 19^{+} \mathrm{Bc}(>2 \%)$ or a high proportion of $\mathrm{CD} 4{ }^{+} \mathrm{Tc}$
$(>15 \%)$ in the IEL preparation after DTT/EDTA treatment is suggestive of contamination with lamina propria lymphocytes, and such cases were not included in our study. Figure 3a illustrates the relatively low proportions of $\mathrm{CD} 103^{-}, \mathrm{CD}_{19}{ }^{+}$and $\mathrm{CD}^{+}$cells in a representative sample prior to magnetic separation. After Annexin-bead separation

a

BEFORE ANNEXIN-BEAD PURIFICATION
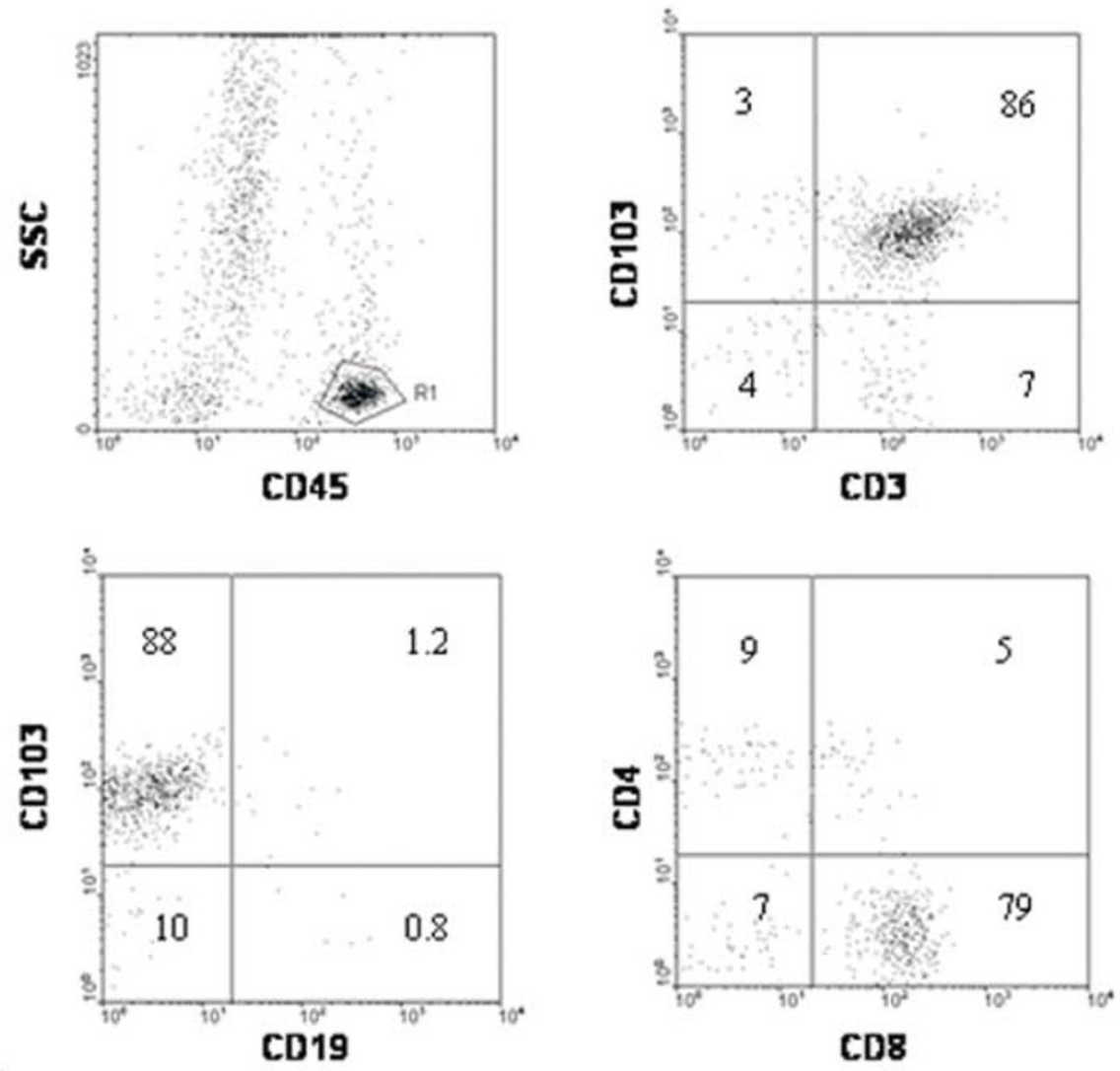

b

\section{AFTER PURIFICATION}
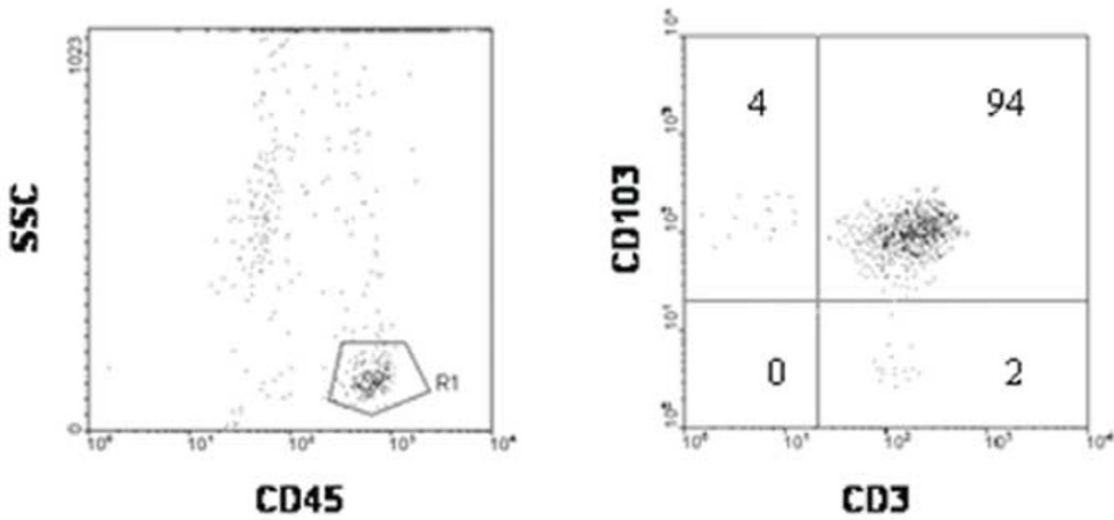

Figure 3 Phenotype of IEL prior to and after Annexin bead separation. IEL were gated on the basis of their low $90^{\circ}$ light scattering (SSC) and high expression of CD45, and their phenotype was studied. In panel (a) phenotype of IEL after Percoll gradient (fraction 40-53\%, note the enrichment for IEL) and prior to Annexin-bead separation. The majority of IEL are CD $103{ }^{+} \mathrm{CD} 3{ }^{+} \mathrm{CD} 8{ }^{+}$cells, as expected. In panel (b) phenotype after a round of Annexin-bead separation, showing the enrichment for CD3 ${ }^{+}$IEL. Shown is an ileal sample, representative of four specimens. 


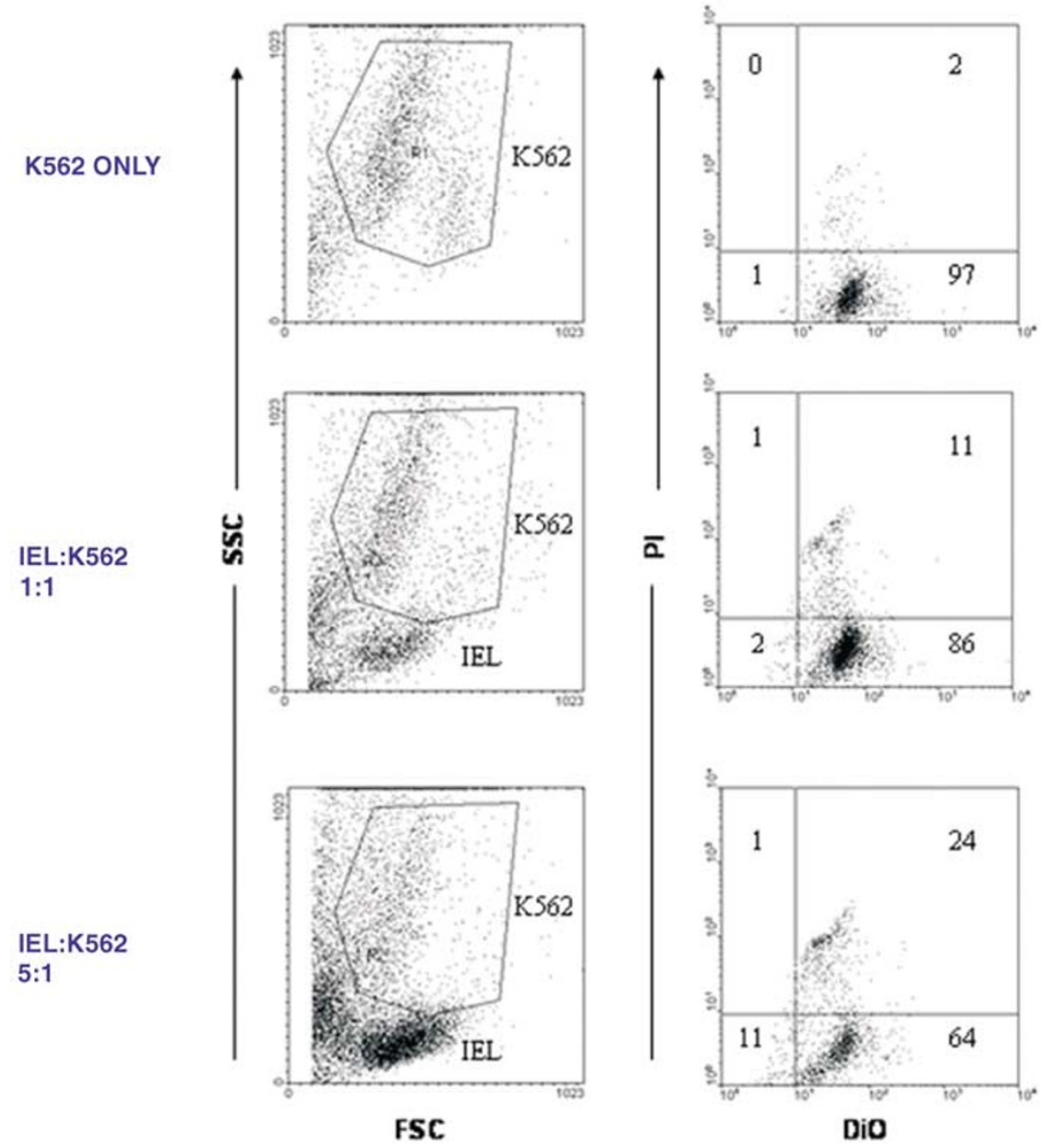

Figure 4 Functionality of isolated IEL: CTL assay. A classic CTL assay was performed by coincubating $\mathrm{DiO}^{+} \mathrm{K}^{2} 62$ target cells and purified IEL for $4 \mathrm{~h}$. The IEL were $>95 \% \mathrm{CD}^{+}$in this representative experiment of 2, performed with ileal IEL. K562 cells were gated based upon their FSC/SSC (left column of dot plots) and the subsequent analysis of PI incorporation was done on $\mathrm{DiO}^{+}$cells to exclude contaminating IEL (right column of dot plots). The upper right dot plot shows the basal PI incorporation of K562 in the absence of IEL. Increasing ratios of effector IEL vs target K562 cells implied increasing incorporation of PI by K562, a sign of their IEL-induced death.

(Figure 3b), we observed a decrease in the proportions of NK IEL (from $10 \pm 5$ to $5 \pm 3 \%$ ), which may possibly be attributed to their inferior viability due to their superior requirement of IEC-produced cytokines such as IL-7 and IL-15. ${ }^{15,16}$

In order to test the functional capacity of isolated IEL, we performed cytotoxicity assays against the classic target cell line K562, previously stained with the marker DiO so they could be identified after coculture with IEL. As it can be observed in Figure 4, the incorporation of propidium iodide (PI) by K562 cells, a sign of cell death, was enhanced in a dose-dependent fashion by coculture with viable purified IEL. This type of cytolysis is mediated by perforins produced by IEL after contact with $\mathrm{K} 562^{6,13}$ and constitutes an indication of the functional capacity of IEL.

In conclusion, in this novel application of Annexin-coated beads, we have obtained highly purified human IEL by taking advantage of the intense externalization of phosphatidyl-serine by human IEC after the conventional IEL isolation process. This rapid and simple method is particularly suitable for functional analyses since it eliminates non viable IEL. This procedure may thus be applied to investigate the role of IEL in intestinal immune responses and to facilitate the diagnosis of lymphoid malignancies in the gut.

\section{Acknowledgements}

This work has been supported by the Spanish Fondo de Investigación Sanitaria (FIS), Grants no 00/0196 and no 01/9417. We thank Drs Camarero, ArgüellesGrande and Quijano for their collaboration in the collection of the mucosal specimens.

\section{References}

1 Cerf-Bensussan N, Guy-Grand D, Griscelli C. Intraepithelial lymphocytes of human gut: isolation, 
characterisation and study of natural killer activity. Gut 1985;26:81-88.

2 Jarry A, Cerf-Bensussan N, Brousse N, et al. Subsets of CD3+ (T cell receptor $\alpha \beta$ and $\gamma \delta$ ) and CD3- lymphocytes isolated from normal human gut epithelium display phenotypic features different from their counterparts in peripheral blood. Eur J Immunol 1990;20: 1097-1103.

3 Sim GK. Intraepithelial lymphocytes and the immune system. Adv Immunol 1995;58:297-343.

4 Camarero C, Eiras P, Asensio A, et al. Intraepithelial lymphocytes and coeliac disease: permanent changes in CD3-/CD7+ and T cell receptor gammadelta subsets studied by flow cytometry. Acta Paediatr 2000;89: 285-290.

5 Fasano A, Berti I, Gerarduzzi T, et al. Prevalence of celiac disease in at-risk and not-at-risk groups in the United States: a large multicenter study. Arch Intern Med 2003;163:286-292.

6 Mention JJ, Ben Ahmed M, Begue B, et al. Interleukin 15: a key to disrupted intraepithelial lymphocyte homeostasis and lymphomagenesis in celiac disease. Gastroenterology 2003;125:730-745.

7 Lundqvist C, Hammarström M-L, Athlin L, et al. Isolation of functionally active intraepithelial lymphocytes and enterocytes from human small and large intestine. J Immunol Methods 1992;152: 253-263.

8 Madrigal L, Lynch S, Feighery C, et al. Flow cytometry analysis of surface major histocompatibility complex class II expression on human epithelial cells prepared from small intestinal biopsies. J Immunol Methods 1993;158:207-214.

9 Eiras P, Leon F, Camarero C, et al. Intestinal intraepithelial lymphocytes contain a CD3-CD7+ subset expressing natural killer markers and a singular pattern of adhesion molecules. Scand J Immunol 2000;52:1-6.

10 Kearsey JA, Stadnyk AW. Isolation and characterization of highly purified rat intestinal intraepithelial lymphocytes. J Immunol Methods 1996;194:35-48.

11 Todd D, Singh AJ, Greiner DL, et al. A new isolation method for rat intraepithelial lymphocytes. J Immunol Methods 1999;224:111-127.

12 Chang L, Gusewitch GA, Chritton DBW, et al. Rapid flow cytometric analysis for the assessment of natural killer cell activity. J Immunol Meth 1993;166:45-54.

13 Leon F, Roldan E, Sanchez L, et al. Human small intestinal epithelium contains functional natural killer lymphocytes. Gastroenterology 2003;125:345-356.

14 Eiras P, Roldan E, Camarero C, et al. Flow cytometry description of a novel CD3-/CD7+ intraepithelia lymphocyte subset in human duodenal biopsies: potential diagnostic value in coeliac disease. Cytometry 1998;34:95-102.

15 Ebert EC. Interleukin 15 is a potent stimulant of intraepithelial lymphocytes. Gastroenterology 1998; 115:1439-1445.

16 Yada S, Nukina H, Kishihara K, et al. IL-7 prevents both caspase-dependent and -independent pathways that lead to the spontaneous apoptosis of i-IEL. Cell Immunol 2001;208:88-95. 